\title{
An analytical study on the boundary size effect of calibration chamber studies
}

\author{
Cheng, Yan ${ }^{\text {i) }}$, Yang, Hongwei ${ }^{\text {ii) }}$ and Sun, De'an ${ }^{\text {iii) }}$ \\ i) Post-doc, Institute for Geology, Mineralogy, and Geophysics, Ruhr-University Bochum, Germany. \\ ii) Post-doc, Department of Civil Engineering, The University of Hong Kong, Pokfulam Road, Hong Kong, China. \\ iii) Professor, Department of Civil Engineering, Shanghai University, 149 Yanchang Road, Shanghai 200072, China.
}

\begin{abstract}
Calibration chambers can provide a laboratory-controlled environment and have long been used to study, such as cone penetration tests and pressuremeter tests. However, the finite radial size of the sample that the chamber can accommodate may give rise to the boundary size effect which can influence the test results. When unsaturated soils, especially for soils where suction hardening is present, are concerned, the effect of suction on the boundary size effect is unknown. This study presents a new cavity expansion solution in unsaturated soils of finite radial extent, with which the boundary size effect can be quantified. The solution is formulated for spherical cavities subjected to two boundary conditions under the drainage condition where the contribution of suction to the effective stress is constant. It is found that samples with a higher suction value are under more influence of the boundary size effect and this is more obvious with higher mean net stress and higher void ratio.
\end{abstract}

Keywords: cavity expansion, boundary size effect, suction, finite radial extent

\section{INTRODUCTION}

Calibration chambers have long been used to develop correlations for interpretation and validate theoretical analysis of cone penetration tests (CPTs) and pressuremeter tests (PMTs) as the stress and strain histories, boundary condition, density and water content of the test samples can be fully controlled. However, due to the limited radial extent of tested samples, the results measured in calibration chambers for unsaturated soil samples may be remarkably different to the values measured in the field, especially for dense samples. As the experiment is analogous to a cavity expanding in finite radial extent, there have been a few attempts (e.g. Yu, 1992, 1993; Salgado et al., 1997; Pournaghiazar et al., 2013a) to solve this problem, though all are limited to saturated soils. Recently, Cheng et al. (2018) presented a cavity expansion solution for unsaturated soils for which suction hardening is present. Here the focus is given to the boundary size effect with insights gained from the new spherical cavity expansion solution for unsaturated soils.

\section{SOIL WATER CHARACTERISTIC CURVE}

The relationship between void ratio $(e)$, degree of saturation $\left(S_{\mathrm{r}}\right)$ and suction $(s)$ is defined through a soil-water characteristic curve (SWCC). A simplified model of Russell and Buzzi (2012) is used where hydraulic hysteresis is ignored. It comprises a main drying curve and an initial transition line between the air expulsion point and the main drying curve (hereafter referred to transition line) (Figure 1):

$$
S_{\mathrm{r}}=\left\{\begin{array}{c}
1 \\
\left(s / s_{\mathrm{ex}}\right)^{\beta} \\
\left(s / s_{\mathrm{ae}}\right)^{\alpha}
\end{array}\right.
$$$$
\begin{aligned}
& \text { for } s \leq s_{\mathrm{ex}} \\
& \text { for } C_{2}^{[\alpha /(\alpha-\beta)]} s_{\mathrm{ex}} \geq s \geq s_{\mathrm{ex}}(1) \\
& \text { for } s \geq C_{2}^{[\alpha /(\alpha-\beta)]} s_{\mathrm{ex}}
\end{aligned}
$$

where $s_{\mathrm{ae}}$ and $s_{\mathrm{ex}}$ are the air entry value and air expulsion value. $\alpha$ is a negative constant. $\beta$ is the slope of the transition line between $s_{\mathrm{ex}}$ and the main drying curve.

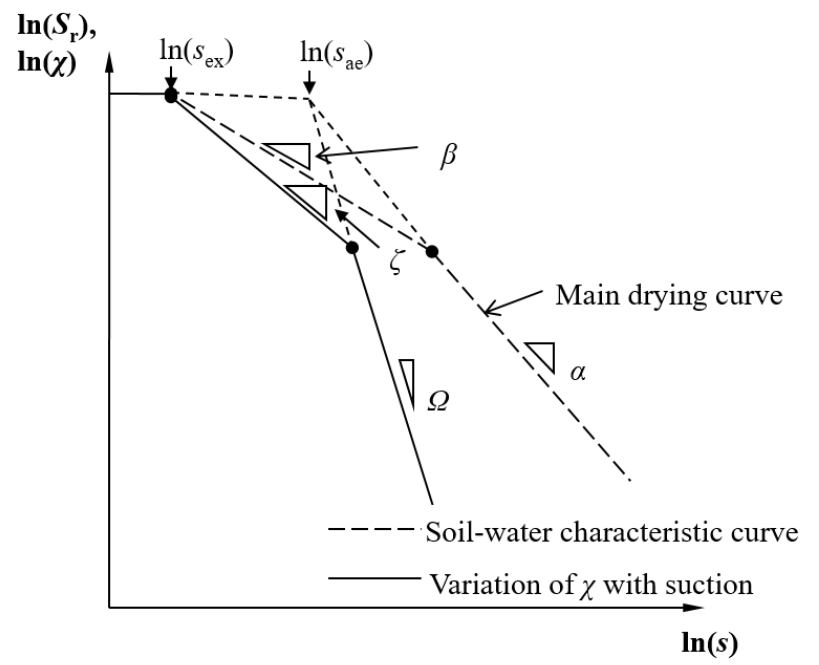

Figure 1. Soil-water characteristic curve and the effective stress parameter $\chi$ plotted against suction. (Extended dashed lines indicate the value where air entries.) 
$s_{\text {ae }}$ depends on void ratio $e$ through

$$
S_{\text {ae }}=C_{1} e^{-\gamma}
$$

in which $C_{1}$ is a positive constant with units of stress and $\gamma$ is a constant. A fixed ratio between $s_{\text {ae }}$ and $s_{\mathrm{ex}}$ is assumed,

$$
s_{\mathrm{ae}}=C_{2} s_{\mathrm{ex}}
$$

in which $C_{2}$ is a constant. For fractal soils $\gamma=D_{s}$, where $D_{s}$ is the fractal dimension of the particle size distribution, and $\alpha=D_{\mathrm{p}}-3$, where $D_{\mathrm{p}}$ is the fractal dimension of the pore size distribution (Russell 2014).

\section{CONSTITUTIVE MODEL}

The stress-strain behavior is described by a simplified version of the bounding surface plasticity model used in Yang and Russell (2015). Conventional $p^{\prime} \sim q$ notation is used, where $p^{\prime}$ and $q$ is the mean effective stress and the deviator stress, respectively. The corresponding strain variables are the volumetric strain $\varepsilon_{\mathrm{p}}$ and shear strain $\varepsilon_{\mathrm{q}}$. Compressive stresses and strains are assumed positive and $\varepsilon_{\mathrm{p}}$ is linked to specific volume $v$ according to $\varepsilon_{\mathrm{p}}=-\ln \left(v / v_{0}\right)$ and $\dot{\varepsilon}_{\mathrm{p}}=-\dot{v} / v$, where $v=1+e$ and $v_{0}$ is the specific volume at the reference configuration. A superimposed dot indicates an increment. Elastic and plastic strain increments sum to give total strain increments and superscripts e and p denote the elastic and plastic components. The stresses and strains are written in vector form, $\boldsymbol{\sigma}^{\prime}=\left[p^{\prime}, q\right]^{\mathrm{T}}$ and $\boldsymbol{\varepsilon}=\left[\varepsilon_{\mathrm{p}}, \varepsilon_{\mathrm{q}}\right]^{\mathrm{T}}$.

The effective stress is:

$$
p^{\prime}=p_{\text {net }}+\chi s \quad \dot{p}^{\prime}=\dot{p}_{\text {net }}+\psi \dot{s}
$$

where $p_{\text {net }}=p-u_{\mathrm{a}}$ is the net stress, $s=u_{\mathrm{a}}-u_{\mathrm{w}}\left(u_{\mathrm{a}}\right.$ and $u_{\mathrm{w}}$ are pore air and water pressures), $\chi$ is the effective stress parameter (having a value of 1 for saturated soils and 0 for dry soils) and $\psi=\mathrm{d}(\chi s) / \mathrm{d} s$. The equation for $\chi$ proposed by Khalili and Khabbaz (1998) is adopted here as:

$$
\chi=\left\{\begin{array}{cc}
1 & \text { for } s \leq s_{\text {ex }} \\
\left(s / s_{\text {ex }}\right)^{-\zeta} & \text { for } C_{2}^{[\Omega /(\Omega-\zeta)]} s_{\text {ex }} \geq s \geq s_{\text {ex }} \\
\left(s / s_{\text {ae }}\right)^{-\Omega} & \text { for } s \geq C_{2}^{[\Omega /(\Omega-\zeta)]} s_{\text {ex }}
\end{array}\right.
$$

where $\Omega$ is a parameter with a best fit value of 0.55 . , $\zeta$ is the slope of the transition line between the $s_{\mathrm{ex}}$ and main drying curve in a $\ln \chi \sim \ln s$ plane. Figure 1 plots the effective stress parameter against suction in the $\ln \chi$ $\sim \ln s$ plane.

A simple isotropic elastic rule is adopted. $K$ and $G$ are defined as:

$$
K=\frac{(1+e) p^{\prime}}{e \kappa} G=\frac{3(1-2 \mu)}{2(1+\mu)} K
$$

where $\kappa$ is a material constant and is the slope of the elastic unload-reload line in the $\ln e \sim \ln p^{\prime}$ plane and $\mu$ is Poisson's ratio. The incremental elastic deformation is given by $\dot{\varepsilon}_{p}^{e}=\dot{p}^{\prime} / K$ and $\dot{\varepsilon}_{q}^{e}=\dot{q} / 3 G$.

The yield surface is defined as

$$
f=q-M_{c s} p^{\prime}\left[\frac{\ln \left(p_{c}^{\prime} / p^{\prime}\right)}{\ln P}\right]^{1 / Q}=0
$$

where $Q$ is a material constant controlling the curvature, $P$ is another material constant controlling the ratio between $p_{c}^{\prime}$ (pre-consolidation pressure) and the value of $p^{\prime}$ at the intercept of $f$ with the critical state line in the $p^{\prime} \sim q$ plane.

Linear critical state lines (CSLs) and limiting isotropic compression lines (LICLs) are assumed in lne $\sim \ln p^{\prime}$ plane. The LICLs depend on suction:

$$
\ln e_{\text {LCL }}=\ln N(s)-\lambda(s) \ln p^{\prime}
$$

where, for a given $s, N(s)$ is the value of $e$ at $\ln p^{\prime}=0$ and $\lambda(s)$ is the slope.

The CSLs are deduced implicitly by an elastic shift from the LICLs along the $\kappa$ line of magnitude $\kappa \ln R$. They are also suction dependent:

$$
\ln e_{\mathrm{CSL}}=\ln N(s)-\lambda(s) \ln \left(R p^{\prime}\right)+\kappa \ln R
$$

A non-associated flow rule is assumed:

$$
d=\frac{\dot{\varepsilon}_{p}^{p}}{\dot{\varepsilon}_{q}^{p}}=A\left(M_{\mathrm{cs}}-\frac{q}{p^{\prime}}\right)
$$

where $d$ is dilatancy and $A$ is a material constant.

The unit vector of plastic flow (m) at $\boldsymbol{\sigma}^{\prime}$ is then:

$$
\mathbf{m}=\left[m_{\mathrm{p}}, m_{\mathrm{q}}\right]^{\mathrm{T}}=\left[\begin{array}{ll}
\frac{d}{\sqrt{1+d^{2}}} & \frac{1}{\sqrt{1+d^{2}}}
\end{array}\right]^{\mathrm{T}}
$$

Imposing the consistency condition at the bounding surface under isotropic hardening leads to:

$$
h=-\frac{\partial f}{\partial p^{\prime}{ }_{c}} \frac{\partial p_{c}^{\prime}}{\partial \varepsilon_{p}^{p}} \frac{m_{p}}{\sqrt{\left(\frac{\partial f}{\partial p^{\prime}}\right)^{2}+\left(\frac{\partial f}{\partial q}\right)^{2}+\left(\frac{\partial f}{\partial p^{\prime}{ }_{c}} \frac{\partial p_{c}^{\prime}}{\partial s}\right)^{2}}}
$$

The relationship between $p_{\mathrm{c}}^{\prime}, s$ and $e$ is:

$$
\frac{\dot{e}}{e}=\frac{\dot{N}(s)}{N(s)}-\dot{\lambda}(s) \ln \left(\bar{p}^{\prime}{ }_{\mathrm{c}}\right)-\lambda(s) \frac{\dot{\bar{p}}^{\prime}{ }_{\mathrm{c}}}{\bar{p}_{\mathrm{c}}{ }_{\mathrm{c}}}
$$

The rate form of the elastic volumetric strain is: 


$$
\dot{\varepsilon}_{\mathrm{p}}^{\mathrm{e}}=\frac{e}{1+e} \kappa \frac{\dot{p}^{\prime}}{p^{\prime}}
$$

The rate form of $p_{\mathrm{c}}^{\prime}$ is:

$$
\begin{aligned}
& p_{\mathrm{c}}^{\prime}=\frac{p_{\mathrm{c}}^{\prime} v}{\lambda(s)-\kappa} \frac{1}{v-1} \dot{\varepsilon}_{\mathrm{p}}^{\mathrm{p}}+ \\
& \frac{p_{\mathrm{c}}^{\prime}}{\lambda(s)-\kappa}\left(\frac{1}{N(s)} \frac{\partial N(s)}{\partial s}-\ln \left(p_{{ }_{\mathrm{c}}}^{\prime}\right) \frac{\partial \lambda(s)}{\partial s}\right) \dot{s}
\end{aligned}
$$

$\mathbf{n}$ defines the direction of loading and is:

$\mathbf{n}=\left[n_{p}, n_{q}, n\right]^{\top}=$

$\left[\begin{array}{c}\frac{\frac{\partial f}{\partial p^{\prime}}}{\sqrt{\left(\frac{\partial f}{\partial p^{\prime}}\right)^{2}+\left(\frac{\partial f}{\partial q}\right)^{2}+\left(\frac{\partial f}{\partial p^{\prime}} \frac{\partial p_{c}^{\prime}}{\partial s}\right)^{2}}}, \frac{\frac{\partial f}{\partial q}}{\sqrt{\left(\frac{\partial f}{\partial p^{\prime}}\right)^{2}+\left(\frac{\partial f}{\partial q}\right)^{2}+\left(\frac{\partial f}{\partial p^{\prime}} \frac{\partial p_{c}^{\prime}}{\partial s}\right)^{2}}} \\ \frac{\frac{\partial f}{\partial p^{\prime}} \frac{\partial p^{\prime}}{\partial s}}{\sqrt{\left(\frac{\partial f}{\partial p^{\prime}}\right)^{2}+\left(\frac{\partial f}{\partial q}\right)^{2}+\left(\frac{\partial f}{\partial p^{\prime},} \frac{\partial p_{c}^{\prime}}{\partial s}\right)^{2}}}\end{array}\right]$

The moisture content $v_{\mathrm{w}}$ can be written in an incremental form $\dot{v}_{\mathrm{w}}=\dot{S}_{\mathrm{r}} e+S_{\mathrm{r}} \dot{e}$. It follows that:

$$
\frac{\partial S_{\mathrm{r}}}{\partial s}(v-1) \dot{s}+S_{\mathrm{r}}(\alpha \gamma+1) \dot{v}=\dot{v}_{\mathrm{w}}
$$

where $\partial S_{\mathrm{r}} / \partial s=\alpha S_{\mathrm{r}} / s$ when the state is on main wetting or drying curves and $\partial S_{\mathrm{r}} / \partial s=\beta S_{\mathrm{r}} / s$ when the state is on a scanning curve.

The complete stress-strain relationship is:

$$
\left[\begin{array}{c}
\dot{\varepsilon}_{\mathrm{p}} \\
\dot{\varepsilon}_{\mathrm{q}} \\
\dot{v}_{\mathrm{w}}
\end{array}\right]=\left[\begin{array}{ccc}
D_{11} & D_{12} & D_{13} \\
D_{21} & D_{22} & D_{23} \\
D_{31} & D_{32} & D_{33}
\end{array}\right]\left[\begin{array}{c}
\dot{p}^{\prime} \\
\dot{q} \\
\dot{s}
\end{array}\right]
$$

where

$$
\begin{aligned}
& D_{11}=\frac{1}{K}+\frac{n_{\mathrm{p}} m_{\mathrm{p}}}{h} \quad D_{12}=\frac{n_{\mathrm{q}} m_{\mathrm{p}}}{h} \quad D_{13}=\frac{n_{\mathrm{s}} m_{\mathrm{p}}}{h} \\
& D_{21}=\frac{n_{\mathrm{p}} m_{\mathrm{q}}}{h} \quad D_{22}=\frac{1}{3 G}+\frac{n_{\mathrm{q}} m_{\mathrm{q}}}{h} \quad D_{23}=\frac{n_{\mathrm{s}} m_{\mathrm{q}}}{h} \\
& D_{31}=-v S_{\mathrm{r}}(\alpha \gamma+1)\left(\frac{1}{K}+\frac{n_{\mathrm{p}} m_{\mathrm{p}}}{h}\right) \quad D_{32}=-v S_{\mathrm{r}}(\alpha \gamma+1) \frac{n_{\mathrm{q}} m_{\mathrm{p}}}{h} \\
& D_{33}=-v S_{\mathrm{r}}(\alpha \gamma+1)\left[\frac{n_{\mathrm{s}} m_{\mathrm{p}}}{h}+\frac{\partial S_{\mathrm{r}}}{\partial s}(v-1)\right]
\end{aligned}
$$

\section{CAVITY EXPANSION ANALYSIS}

A homogenous soil mass with radius $B$ is initially under a mean effective stress $p^{\prime}{ }_{0}$. A concentric cavity is then expanded from its initial radius $a_{0}$ to $a$, and a region consisting of elastic and plastic strains is formed around the cavity with the radius of the elastic-plastic boundary $R$. The elastic-plastic region is surrounded by soil which deforms purely elastically and has boundary $B$. The configuration is illustrated in Figure 2, where the radius of a particular soil element is denoted by $r$.

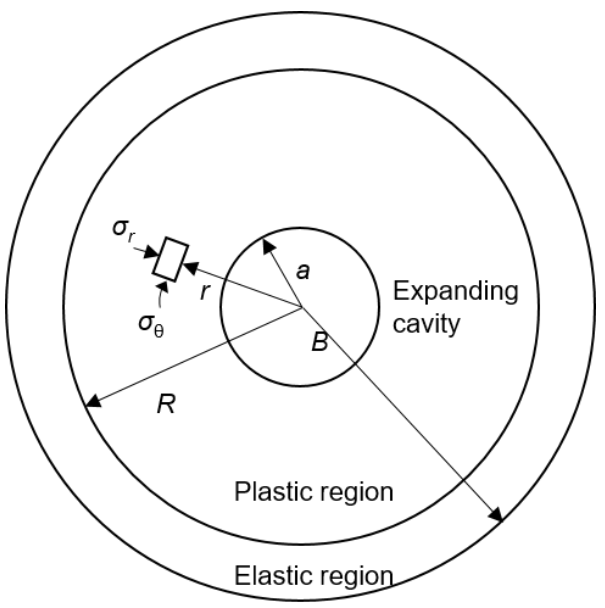

Figure 2. Configuration of the cavity expansion problem

The radial stress $\left(\sigma_{\mathrm{r}}^{\prime}\right)$ represents the major principal stress and the tangential stress $\left(\sigma_{\theta}^{\prime}\right)$ represents the minor principal stress. $p^{\prime}$ and $q$ are defined in terms of $\sigma_{\mathrm{r}}^{\prime}$ and $\sigma_{\theta}^{\prime} . \varepsilon_{\mathrm{p}}$ and $\varepsilon_{\mathrm{q}}$ are related to radial strain $\varepsilon_{\mathrm{r}}$ and tangential strain $\varepsilon \theta$.

$p^{\prime}=\frac{\sigma_{\mathrm{r}}^{\prime}+2 \sigma_{\theta}^{\prime}}{3}, q=\sigma_{\mathrm{r}}^{\prime}-\sigma_{\theta}^{\prime}, \varepsilon_{\mathrm{p}}=\varepsilon_{\mathrm{r}}+2 \varepsilon_{\theta}, \varepsilon_{\mathrm{q}}=\frac{2\left(\varepsilon_{\mathrm{r}}-\varepsilon_{\theta}\right)}{3}$

$\varepsilon_{\mathrm{r}}$ and $\varepsilon_{\theta}$ at $r$ are defined in terms of radial displacement $u$ of the soil element at $r$ by $\varepsilon_{\mathrm{r}}=-\partial u / \partial r$ and $\varepsilon_{\theta}=-u / r$.

\subsection{Finite elastic region}

The solution for stress distribution and displacement in an elastic region for $R \leq r \leq B$ is given as follows. For constant stress boundary condition, the stress and displacement distributions are:

$$
\begin{aligned}
& \sigma_{r}^{\prime}=p_{0}{ }_{0}+\left(\sigma_{R}^{\prime}-p_{0}^{\prime}\right)\left[\frac{(R / r)^{3}-(R / B)^{3}}{1-(R / B)^{3}}\right] \\
& \sigma_{\theta}^{\prime}=p_{0}^{\prime}-\left(\sigma_{R}^{\prime}-p_{0}^{\prime}\right)\left[\frac{\frac{1}{2}(R / r)^{3}+(R / B)^{3}}{1-(R / B)^{3}}\right]
\end{aligned}
$$




$$
\begin{aligned}
& u=\frac{\sigma_{R}^{\prime}-p_{0}^{\prime}}{\left(1 /{ }_{R}\right)^{3}-\left(1 /{ }_{B}\right)^{3}} \frac{1}{2 G(1+\mu)} \\
& \left(\frac{1+\mu}{k r^{2}}+\frac{1-2 \mu}{B^{3}} r\right)
\end{aligned}
$$

For the zero displacement boundary condition, the solutions for stress and displacement in an elastic region for $R \leq r \leq B$ are:

$$
\begin{aligned}
& \sigma_{r}^{\prime}=p_{0}^{\prime}+ \\
& \left(\sigma_{R}^{\prime}-p_{0}^{\prime}\right)\left[\frac{\left(R /{ }^{3}+\frac{1+\mu}{2(1-2 \mu)}\left(R /{ }^{3}\right)^{3}\right.}{\frac{1+\mu}{2(1-2 \mu)}\left(R /{ }_{B}\right)^{3}+1}\right] \\
& \sigma_{\theta}^{\prime}=p_{0}^{\prime}- \\
& \left(\sigma_{R}^{\prime}-p_{0}^{\prime}\right)\left[\frac{\frac{1}{2}(R / r)^{3}-\frac{1+\mu}{2(1-2 \mu)}(R / B)^{3}}{\frac{1+\mu}{2(1-2 \mu)}(R / B)^{3}+1}\right] \\
& u=\left(\sigma_{R}^{\prime}-p_{0}^{\prime}\right) r \frac{1}{4 G(1+\mu)} \\
& \left\{\begin{array}{c}
(1+\mu)(R / r)^{3}-(1+\mu)(R / B)^{3} \\
\frac{1+\mu}{2(1-2 \mu)}(R / B)^{3}+1
\end{array}\right\}
\end{aligned}
$$

where $G$ is the elastic shear modulus and defines as $E / 2(1+\mu)$.

\subsection{Plastic region}

Seven differential equations govern soil behavior in the plastic region. The seven equations involve seven variables and can be presented in differential form and solved as an initial value problem.

The stress equilibrium equation is:

$$
\frac{\partial p^{\prime}}{\partial r}+\frac{2}{3} \frac{\partial q}{\partial r}+\frac{2 q}{r}=0
$$

The incremental forms of plastic strains come directly from the bounding surface model:

$$
\mathrm{D} \varepsilon_{p}^{p}=\mathrm{D} \varepsilon_{p}-\mathrm{D} \varepsilon_{p}{ }^{e}=\frac{1}{h}\left(n_{\mathrm{p}} \mathrm{D} p^{\prime}+n_{\mathrm{q}} \mathrm{D} q+n_{\mathrm{s}} \mathrm{D} s\right) m_{\mathrm{p}}
$$

$$
\mathrm{D} \varepsilon_{q}^{p}=\mathrm{D} \varepsilon_{q}-\mathrm{D} \varepsilon_{q}{ }^{e}=\frac{1}{h}\left(n_{\mathrm{p}} \mathrm{D} p^{\prime}+n_{\mathrm{q}} \mathrm{D} q+n_{\mathrm{s}} \mathrm{D} s\right) m_{\mathrm{q}}
$$

The incremental relationship between moisture content, suction and specific volume is:

$$
\frac{\partial S_{r}}{\partial s}(v-1) \mathrm{D} s+S_{r}(\alpha \gamma+1) \mathrm{D} v=\mathrm{D} v_{w}
$$

The rate forms for $s_{\mathrm{ae}}$ is

$$
\mathrm{D} s_{a e}+C_{1} \gamma(v-1)^{-\gamma-1} \mathrm{D} v=0
$$

Suction has a multiplicative effect on hardening:

$$
\begin{aligned}
& \mathrm{D} p_{c}^{\prime}=-\frac{p_{c}^{\prime}}{\lambda(\mathrm{s})-\kappa} \frac{1}{v-1} \mathrm{D} v-\frac{v}{K} \frac{p_{{ }_{c}}}{\lambda(\mathrm{s})-\kappa} \frac{1}{v-1} \mathrm{D} p^{\prime} \\
& +\frac{p_{{ }_{c}}^{\prime}}{\lambda(\mathrm{s})-\kappa}\left(\frac{\partial N(\mathrm{~s})}{\partial s}-\ln \left(p_{c}^{\prime}\right) \frac{\partial \lambda(\mathrm{s})}{\partial s}\right) \mathrm{D} s
\end{aligned}
$$

Taking advantage of the findings of Yang and Russell (2015), that constant $\chi s$ can be assumed without loss of significant accuracy, when the actual drainage conditions are different, the constant $\chi s$ condition is assumed here:

$$
\frac{\partial(\chi s)}{\partial r}=\Psi \frac{\partial s}{\partial r}+\chi \Omega \frac{s}{s} \frac{\partial s_{e}}{\partial r}=0
$$

In the elastic region the solution procedure is straightforward and involves using the above closed form expressions for stresses, strains and velocity in terms of $r$ and $R$. In the plastic region the solution procedure is more complex and the governing equations are not described in the same scheme, in that Eqs. (33-38) are formulated at material time whereas Eq. (32) is at local time. The auxiliary independent variable proposed by Chen and Abousleiman (2013) is adopted here to bring these equations into the same scheme, which is defined as:

$$
\xi=\frac{u}{r}=\frac{r-r_{0}}{r}
$$

After some manipulations of $\xi$, the governing Eqs. (32-37) and drainage condition (38) can also be expressed with respect to $\xi$. These governing equations may be presented in matrix form as: 


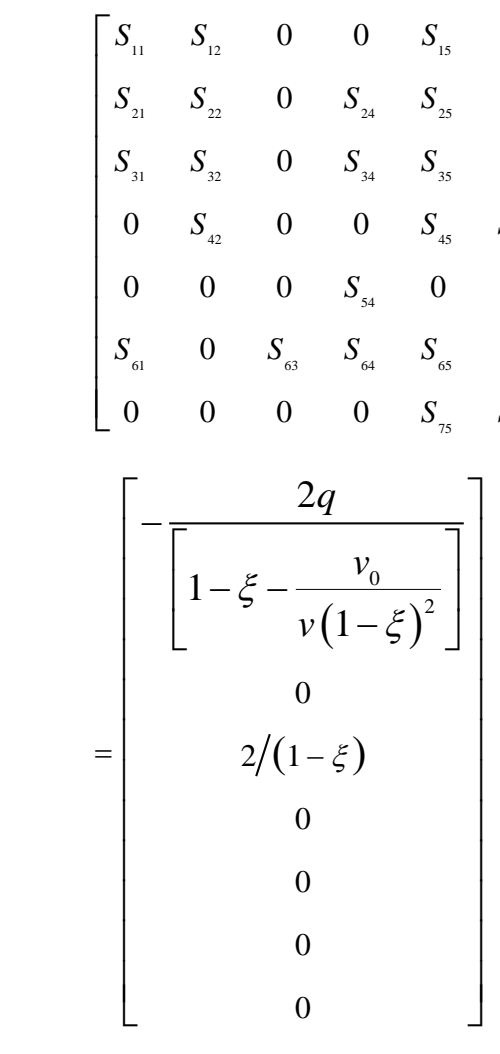$$
\left.\begin{array}{c}
S_{17} \\
0 \\
0 \\
0 \\
S_{57} \\
0 \\
S_{77}
\end{array}\right]\left[\begin{array}{c}
\mathrm{D} p^{\prime} / \mathrm{D} \xi \\
\mathrm{D} q / \mathrm{D} \xi \\
\mathrm{D} p^{\prime} / \mathrm{D} \xi \\
\mathrm{D} v / \mathrm{D} \xi \\
\mathrm{D} s / \mathrm{D} \xi \\
\mathrm{D} v_{w} / \mathrm{D} \xi \\
\mathrm{D}{ }{ } / \mathrm{D} \xi
\end{array}\right]
$$

where

$$
\begin{gathered}
S_{11}=1 \quad S_{12}=\frac{2}{3} S_{15}=-\Psi \quad S_{17}=-\chi \Omega \frac{s}{S_{a e}} \\
S_{21}=\frac{1}{K}+\frac{1}{h} n_{\mathrm{p}} m_{\mathrm{p}} S_{22}=\frac{1}{h} n_{\mathrm{q}} m_{\mathrm{p}} S_{24}=\frac{1}{v} S_{25}=\frac{1}{h} n_{\mathrm{s}} m_{\mathrm{p}} \\
S_{31}=\frac{1}{h} n_{\mathrm{p}} m_{q} \quad S_{32}=\frac{1}{3 G}+\frac{1}{h} n_{\mathrm{q}} m_{q} \quad S_{34}=\frac{2}{3} \frac{1}{v} \\
S_{35}=\frac{1}{h} n_{\mathrm{s}} m \\
S_{42}=S_{r}(\alpha \gamma+1) \quad S_{45}=\frac{\partial S_{r}}{\partial s}(v-1) \\
S_{54}=C_{1} \gamma(v-1)^{-\gamma-1} S_{57}=1 \quad S_{46}=-1 \\
S_{61}=\frac{v}{K} \frac{p_{c}^{\prime}}{\lambda(\mathrm{s})-\kappa} \quad S_{63}=1 \\
S_{65}=-\frac{p_{c}^{\prime}}{\lambda(\mathrm{s})-\kappa_{c}}\left(\frac{\partial N(\mathrm{~s})}{\partial s}-\ln \left(p_{c}^{\prime}\right) \frac{\partial \lambda(\mathrm{s})}{\partial s}\right) \\
S_{75}=\Psi \text { and } S_{77}=\frac{\chi \Omega s}{s_{e}} \quad S_{c}^{\prime}
\end{gathered}
$$

Now the governing differential equations in the plastic region are all ordinary differential equations with respect to $\xi$ and can be solved as initial value problem once the initial conditions at the elastic-plastic boundary are known.

\subsection{Initial conditions and elastic-plastic boundary}

By insisting that the conditions at the elastic-plastic boundary $(r=R)$ must satisfy the governing equations in elastic region (20) and (21) or (23) and (24) (depending on the boundary condition), after expansion of the cavity to a certain radius, the driver starts from $\xi_{\mathrm{p}}$ which for constant stress boundary conditions is:

$$
\begin{aligned}
\xi_{p}=\left(\frac{u}{r}\right)_{r=r_{p}}= & \frac{\sigma_{r, p}^{\prime}-p_{0}^{\prime}}{(1 / R)^{3}-\left(1 / B^{3}\right)^{3}} \frac{1}{2 G(1+\mu)} \\
& {\left[\frac{1+\mu}{2 R^{3}}+\frac{1-2 \mu}{B^{3}}\right] }
\end{aligned}
$$

where $\sigma_{\mathrm{r}, \mathrm{p}}^{\prime}$ is obtained by substituting Eqs. (20) and (21) into $\eta_{\mathrm{p}}$ and rearranging as:

$$
\sigma_{r, p}^{\prime}=p_{0}^{\prime}\left[\frac{1+\frac{2}{3} \eta_{p}}{1+\frac{2}{3}\left(\frac{R}{B}\right)^{3} \eta_{p}}\right]
$$

Similarly for zero displacement boundary conditions:

$$
\begin{gathered}
\xi_{p}=\left(\frac{u}{r}\right)_{r=r_{p}}=\left(\sigma_{r, p}^{\prime}-p_{0}^{\prime}\right) \frac{1}{4 G(1+\mu)} \\
\left\{\frac{1-(R / B)^{3}}{\frac{1}{2(1-2 \mu)}(R / B)^{3}+1}\right\}
\end{gathered}
$$

where $\sigma_{r, p}^{\prime}$ is:

$$
\sigma_{r, p}^{\prime}=p_{0} \cdot\left[\frac{1+\frac{2}{3} \eta_{p}}{1-\frac{1+\mu}{3(1-2 \mu)}(R / B)^{3} \eta_{p}}\right]
$$

To link the auxiliary independent variable with radial coordinate, it has:

$$
\frac{\mathrm{d} r}{r}=\frac{\mathrm{d} \xi}{1-\xi-v_{0} /\left[v(\xi)(1-\xi)^{2}\right]}
$$

Integrating Eq.(39) from the cavity wall to the radial distant to the plastic region, the relation between $r / a$ and $\xi$ can be obtained as: 


$$
\frac{r}{a}=\exp \left(\int_{\xi_{a}}^{\xi} \frac{\mathrm{d} \xi}{1-\xi-v_{0} /\left[v(\xi)(1-\xi)^{2}\right]}\right)
$$

\section{RESULTS AND DISCUSSIONS}

\subsection{Input parameters}

Model parameters for a silty sand from Yang and Russell (2015) are adopted here. The particle size and pore size distributions are fractal with $D_{\mathrm{s}}=2.6$ and $D_{\mathrm{p}}=$ 2.48. Also, $C_{1}=1.5 \mathrm{kPa}$ and $C_{2}=30$. It follows that $s_{\mathrm{ac}}=1.5 e^{-2.61} \mathrm{kPa}, s_{\mathrm{ae}}=30 s_{\mathrm{ex}}, \alpha=-0.52$ and $\gamma=2.61 . \beta=$ -0.2 fits the transition line. As $\Omega=0.55$ it follows that $\zeta$ $=\beta \Omega / \alpha=0.2115$. The elasticity parameters are $\kappa=$ 0.012 and $\mu=0.4$. The critical state strength is $M_{\mathrm{cs}}=$ 1.45 (corresponding to $\left.\phi_{\text {tr }}^{\prime}=35.7^{\circ}\right)$. The parameter $A=$ 1.2 is adopted. $Q=2.8$ and $P=10$ define the shape of the yield surface. $\lambda(s)$ and $N(s)$ for different suction values are listed in Table $1 . \lambda(s)$ and $N(s)$ for intermediate suction values are found by linear interpolation and for suctions larger than $300 \mathrm{kPa}$ by linear extrapolation from the $100 \mathrm{kPa}$ to $300 \mathrm{kPa}$ interval.

Table 1. $\lambda(s)$ and $N(s)$ for different suction values.

\begin{tabular}{ccccc}
\hline$s(\mathrm{kPa})$ & $s \leq s_{\mathrm{ex}}$ & $s=10$ & 100 & 300 \\
\hline$N(s)$ & 0.515 & 1.4765 & 1.58 & 1.81 \\
$\lambda(s)$ & 0.08 & 0.1555 & 0.16 & 0.17 \\
\hline
\end{tabular}

Cavity expansoin results are then generated for a range of initial stress states involving $p_{\text {net } 0}=60 \mathrm{kPa}, 90$ $\mathrm{kPa}, 120 \mathrm{kPa}$ or $240 \mathrm{kPa}, s_{0}=20 \mathrm{kPa}, 80 \mathrm{kPa}$ or $160 \mathrm{kPa}$ and $e_{0}=0.43,0.51,0.58$ or 0.66 . The initial stress states are illustrated in the $\ln e \sim \ln p^{\prime}$ plane together with corresponding CSL and LICL, as shown in Figure 3. The specific volumes selected represent loose, medium and dense state, respectively.

\subsection{Boundary size effect}

To explore the boundary size effect, results are plotted in the $\sigma_{\mathrm{L}} / \sigma_{\mathrm{L}, \infty} \sim B / a$ plane (where $\sigma_{\mathrm{L}, \infty}$ denotes the limiting cavity pressure in infinite radial extent and is obtained when $R / B=0$ ) as shown in Figure 4 for spherical cavity expansion. The ratio of $B / a$ is calculated as the product of $R / a$ at the elastic-plastic boundary and input value of $R / B$. The results for $s_{0}=20$ $\mathrm{kPa}$ and $160 \mathrm{kPa}$ are plotted to highlight the effect of suction.

As shown in Figure 4 for a certain $e_{0}$, the reduction in the $\sigma_{\mathrm{L}}$ under constant stress boundary conditions and the increase in the $\sigma_{\mathrm{L}}$ under zero displacment boundary conditions becomes smaller as $B / a$ increases, as expected. At a certain value of $B / a$, the value of $\sigma_{\mathrm{L}} / \sigma_{\mathrm{L}, \infty}$ at $s_{0}=160 \mathrm{kPa}$ deviates more from unity than that at $s_{0}$
$=20 \mathrm{kPa}$ and this is more obvious with higher values of $p_{\text {neto }}$, which is probably due to the stiffer response at higher $s_{0}$ and $p_{\text {neto }}$. This suggests samples with a higher suction value are under more influence of the boundary size effect, especially at larger initial mean net stresses. The effect is less significant as $e_{0}$ is reduced as the lines for different suction values become more indistinguishable and also the results for different $p_{\text {net0 }}$ prone to converge so that the contribution of suction to $p_{0}^{\prime}$ has less effect on $\sigma_{\mathrm{L}} / \sigma_{\mathrm{L}, \infty}$.

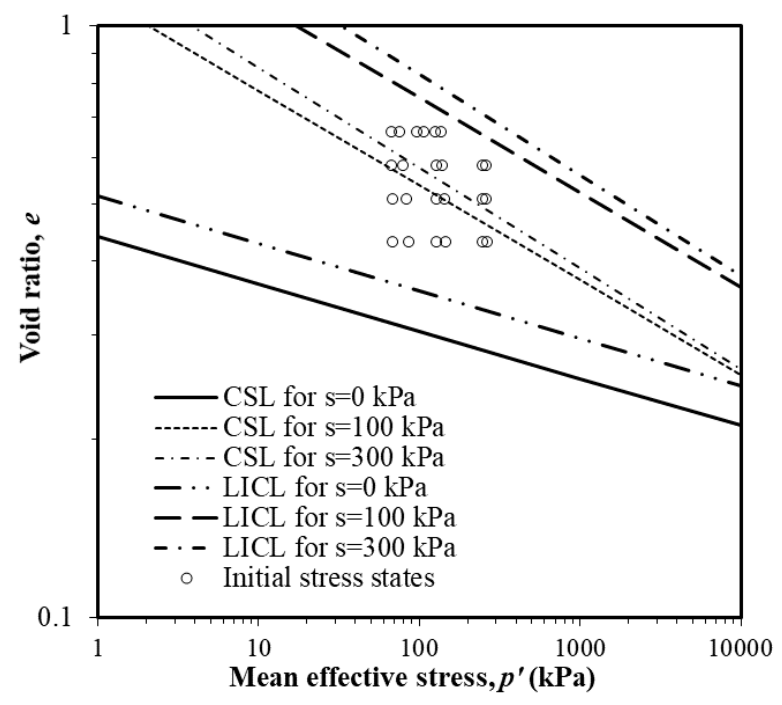

Figure 3. Initial stress states with CSL and LICL lines for the cavity expansion analysis.

The effect of suction on $R / a$ can also be observed in these figures. Taking Figure 4 (b) for example, for constant stress condition, at a certain $R / B$ a larger value of $R / a$ resulting from higher suction is associated with a higher value of $B / a$. And the larger $R / a$ is associated with smaller value of $\sigma_{\mathrm{L}} / \sigma_{\mathrm{L}, \infty}$. The opposite is for zero displacement condition, that higher suction results in a smaller $R / a$ but a higher value of $\sigma_{\mathrm{L}} / \sigma_{\mathrm{L}, \infty^{\circ}}$. Again the effect is more obvious for larger $e_{0}$ and higher $p_{\text {neto }}$.

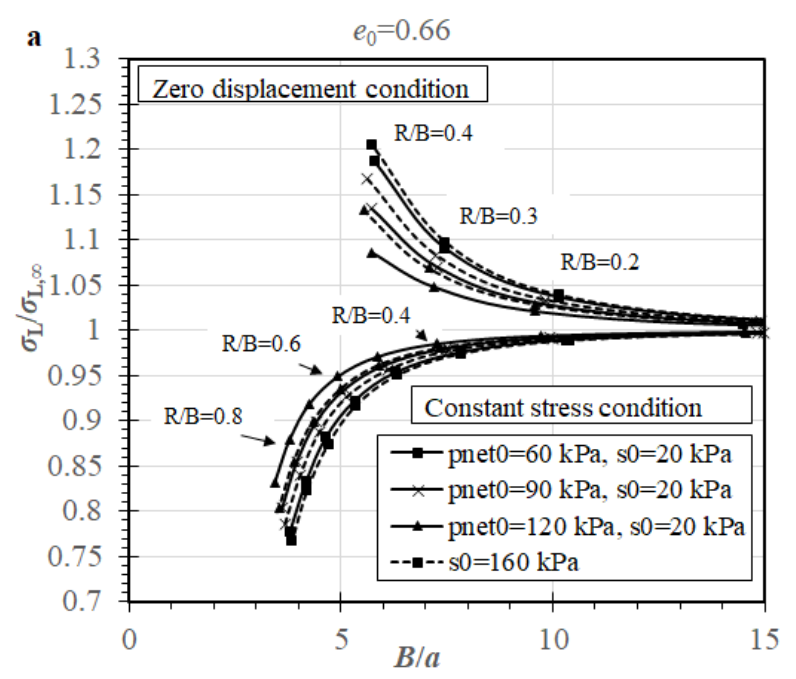



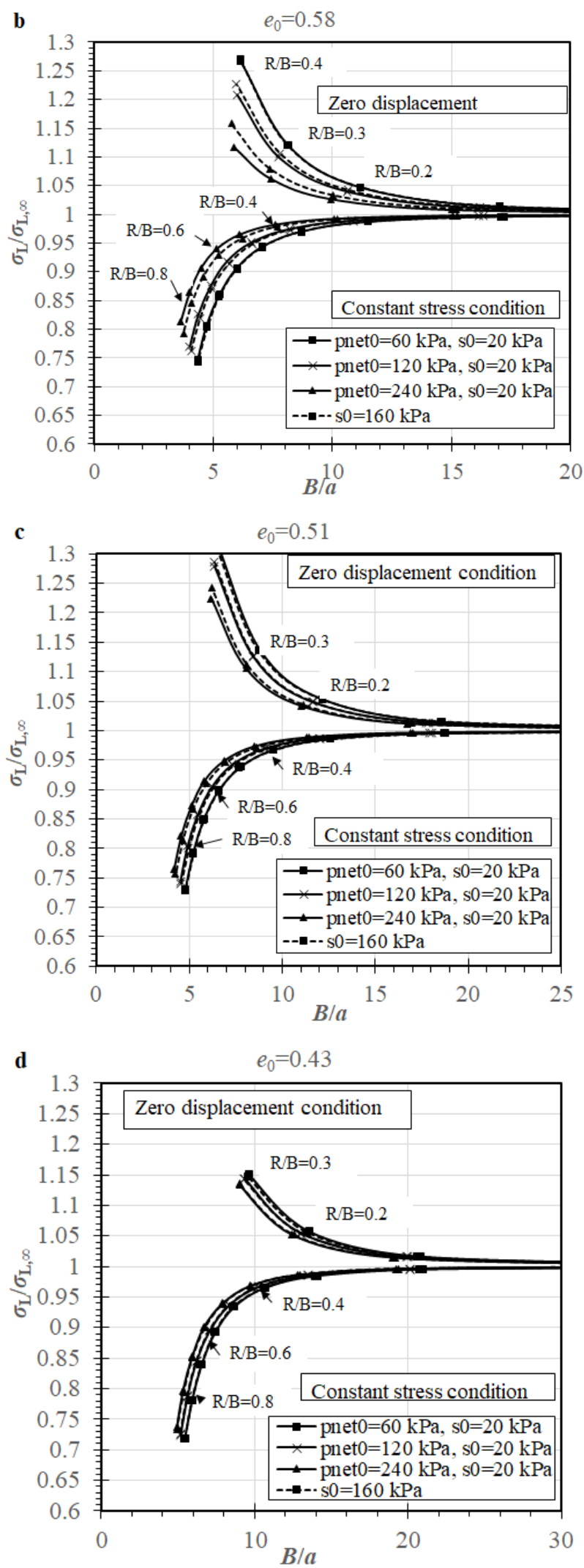

Figure 4. Constant stress and zero displacement boundary effects on spherical cavity expansion results for various initial mean effective stresses, (a) $e_{0}=0.66$, (b) $e_{0}=0.58$, (c) $e_{0}=0.51$ and (d) $e_{0}=0.43$. Dashed lines indicate results at $s_{0}=160 \mathrm{kPa}$.

\section{CONCLUSIONS}

This paper presents a generalized solution for a spherical cavity expanding in unsaturated soils of finite radial extent subjecting to two boundary conditions. The finite radial extent is represented by the value of $R / B$ (radius ratio of plastic region to finite radial extent) which is taken as a shape factor. The effects of boundary conditions under different values of shape factor are reflected by the values at the elastic-plastic boundary and thus the initial values for the solution in the plastic region.

The role of suction on the boundary size effect is examined and is found to be significant, especially at higher initial mean net stress and at higher initial void ratio. This suggests the soil mass with a higher suction value are under more influence of the boundary size effect.

\section{REFERENCES}

1) Chen S.L and Abousleiman Y.N. (2013): Exact drained solution for cylindrical cavity expansion in modified Cam Clay soil. Geotechnique 2013, 63(6), 510-517.

2) Cheng, Y., Yang, HW and Sun DA. (2018): Cavity expansion in unsaturated soils of finite radial extent. Computers and Geotechnics, 102, 216-228.

3) Khalili N. and Khabbaz, M.H. (1998): Unique relationship for $\chi$ for the determination of the shear strength of unsaturated soils. Geotechnique, 48(5), 681-687.

4) Russell AR, Buzzi O. (2012): A fractal basis for soil-water characteristics curves with hydraulic hysteresis. Geotechnique, 62(3), 269-274.

5) Russell AR. (2014): How water retention in fractal soils depends on particle and pore sizes, shapes, volumes and surface areas. Geotechnique, 64(5), 379-90.

6) Salgado R, Mitchell JK, Jamiolkowski M. (1997) Cavity expansion and penetration resistance in sand. $\mathrm{J}$ Geotech Geoenviron, 123(4), 344-54.

7) Pournaghiazar M, Russell AR, Khalili N. (2013): Drained cavity expansions in soils of finite radial extent subjected to two boundary conditions. International Journal for Numerical and Analytical Methods in Geomechanics, 37(4), 331-352

8) Yang, $H$ and Russell,A.R.(2015): Cavity expansion in unsaturated soils exhibiting hydraulic hysteresis considering three drainage conditions. International Journal of Numerical and Analytical Methods in Geomechanics 2015, 39, 1975-2016.

9) Yu, H.S., Schnaid, F. and Collins, I.F. (1996): Analysis of cone pressuremeter tests in sands. Journal of Geotechnical Engineering ASCE, 122(8), 623-632 